\title{
Traitement Chirurgical Des Fractures Du Fémur De L'enfant
}

\author{
Walla A \\ Service de chirurgie orthopédique et traumatologique \\ du CHU Sylvanus Olympio, Lomé, Togo \\ Gnassingbe $\mathrm{K}$ \\ Mihleudo-Agbolan $K$ \\ Zeba ZAA \\ Goudjo E \\ Ndougssa I \\ Akakpo-Numado G K \\ Tekou $\mathrm{H}$
}

Service de chirurgie pédiatrique du CHU Sylvanus Olympio, Lomé, Togo

doi: 10.19044/esj.2016.v12n3p92 URL:http://dx.doi.org/10.19044/esj.2016.v12n3p92

\begin{abstract}
Objective To describe indications, different modalities and results of surgical treatment of femur fractures in children.

Patients and methods This is a retrospective study from January 2004 to December 2013 in the pediatric surgery department of Lomé Sylvanius Olympio teaching hospital, from the records of patients aged 0-15 years with femur fractured treated surgically.

Results There were $66.1 \%$ (39 cases) of shaft fractures of which $53.57 \%$ localized in the middle third. Of the $33.9 \%$ (20 cases) of the lower extremity fracture, epiphyseal growth fractures accounted for 13 cases including seven (7) for type II among Salter-Harris classification. The indications were: femoral fracture occurred in the context of multiple trauma (4 cases), patients over 11 years (40 cases), failure of conservative treatment (2 patients), epiphyseal growth fractures of the distal femur after failure of conservative treatment (12 patients) and an epiphyseal growth fracture type 4 of Salter and Harris where surgery was indicated immediately (1 case). The average time of surgical treatment was 32 days. The elastic stable intramedullary nailing (ESIN) was made in $66.1 \%$ of patients, the plate in $18.6 \%$, the pinning in $11.9 \%$ and screwing in $3.4 \%$. The average duration of hospital stay was 45.16 days. Removal of osteosynthesis material was performed in 38 patients or $64.4 \%$ of cases. The average time of this removal was 8 months.
\end{abstract}


Conclusion The methods of the most widely used surgical treatment are open ESIN because of the long waiting patients associated with very limited financial resources. This method has real advantages and should be encouraged in our communities by improving the technical facilities and living conditions of the population.

Keywords: Femoral fractures, children, elastic stable intramedullary nailing, Togo

\section{Résumé}

Objectif Décrire les indications, les différentes modalités et les résultats du traitement chirurgical des fractures du fémur chez l'enfant.

Patients et methods Il s'agit d'une étude rétrospective effectuée de janvier 2004 à décembre 2013 dans le service de chirurgie pédiatrique du CHU Sylvanus Olympio de Lomé, à partir des dossiers de patients âgés de 0 à 15 ans ayant une fracture du fémur et dont la prise en charge était chirurgicale.

Résultats Il y avait 66,1\% (39 cas) de fractures diaphysaires dont 53,57\% localisées au tiers moyen. Sur les 33,9\% (20 cas) de fracture de l'extrémité inférieure, les fractures décollements épiphysaires ont représenté 13 cas dont sept (7) de type II de Salter et Harris. Les indications étaient : fracture du fémur survenue dans le cadre d'un polytraumatisme (4 cas), patients de plus de 11 ans (40 cas), échec du traitement orthopédique (2 cas), fracture décollement épiphysaire de l’extrémité inférieure du fémur après échec du traitement orthopédique (12 cas) et une fracture décollement épiphysaire type 4 de Salter et Harris où la chirurgie a été d'emblée indiquée (1 cas). Le délai moyen de prise en charge chirurgicale était de 32 jours. L’embrochage centromédullaire élastique stable (ECMES) a été fait chez 66,1\% des patients, la plaque vissée chez 18,6\%, le brochage chez $11,9 \%$ et le vissage chez 3,4\%. La durée moyenne d'hospitalisation était de 45,16 jours. L'ablation du matériel d’ostéosynthèse a été réalisée chez 38 patients soit 64,4\% des cas. Le délai moyen de cette ablation était de 8 mois.

Conclusion Les méthodes de traitement chirurgical les plus employées sont l'ECMES à foyer ouvert en raison de la longue durée d'attente des patients liée aux moyens financiers très limités. Cette méthode présente des avantages certains et sa pratique devra être encouragée dans nos milieux en améliorant le plateau technique et les conditions de vie de la population.

Mots clés : fractures du fémur, enfants, embrochage centromédullaire élastique stable, Togo 


\section{Introduction}

Les fractures du fémur de l'enfant représentent l'un des types de fractures le plus répandu avec une incidence de 1,6\% (ASLANI, 2013). Elles font suite souvent à des accidents de motocyclistes en circulation et affectent beaucoup plus les garçons (CAPRA, 2013).

Réputées bénignes, les fractures du fémur chez le petit enfant, ont longtemps été traitées quasi exclusivement de façon orthopédique (CHIGOT, 1967). Le résultat final est habituellement satisfaisant.

Après l’âge de 6 ans, le traitement est plus controversé (RAMSEIER, 2010). Ces dernières années, la tendance à l'utilisation de différentes méthodes chirurgicales pédiatriques dans les fractures du fémur a largement augmenté (ASLANI, 2013). Cette option permet de réduire les coûts des soins et la durée d'hospitalisation pour répondre aux contraintes de la scolarisation des enfants victimes (AKAKPO-NUMADO, 2014). Plusieurs techniques d'ostéosynthèse ont été proposées: l'embrochage de Hackethal et l'enclouage de Ender (PARSCH, 1997), l’ostéosynthèse par plaque vissée (KREGOR, 1993), l'embrochage centromédullaire élastique stable (LIGIER, 1998) et l'exofixation en cas de fracture ouverte (ASLANI, 2013). Chacune de ces méthodes présente des avantages et inconvénients. Dans notre pratique quotidienne, certaines de ces méthodes chirurgicales sont utilisées dans la prise en charge des patients.

Cette étude a été initiée dans le but de décrire les indications, les différentes modalités et les résultats du traitement chirurgical des fractures du fémur chez l'enfant.

\section{Patients et méthodes}

Il s’agit d'une étude rétrospective effectuée de janvier 2004 à décembre 2013 dans le service de chirurgie pédiatrique du CHU Sylvanius Olympio de Lomé, à partir des dossiers de patients âgés de 0 à 15 ans ayant une fracture du fémur et dont la prise en charge était chirurgicale. Il n'y avait pas de table d'opération orthopédique, ni d'amplificateur de brillance. L’ouverture cutanée a été classée selon Cauchoix et Duparc. Chez 57 patients, la réduction a été faite à foyer ouvert. Le délai de prise en charge est l'intervalle de temps entre la survenue du traumatisme et la réalisation de l'ostéosynthèse. L'ablation du matériel d'ostéosynthèse a été décidée sur les contrôles radiographiques en présence d'un cal unitif.

Il y avait 34 garçons (57,63\%) et 25 filles (42,37\%) soit une sex-ratio (H-F) de 1,36. L'âge moyen des patients était de 11,4 ans avec des extrêmes de 5 ans et 15 ans. Les patients dont l’âge était compris entre 6 et 11 ans avaient représenté près de 51\%. Durant les 10 ans, 696 patients ont été traités pour fracture du fémur dont 59 chirurgicalement, ce qui représente 8,47\% de l'ensemble des fractures du fémur. En cas de brochage en croix, les patients 
ont reçu une immobilisation plâtrée cruro-pédieuse complémentaire pour une durée de 45 jours de même que chez 10 patients traités par ECMES dont le montage a été jugé instable. Les accidents de la voie publique (AVP), (33 ; $55,9 \%)$ et les accidents de jeu $(18 ; 30,5)$ ont été les circonstances de survenue majoritaires.

\section{Conflit d'intérêt : Aucun}

\section{Résultats}

Toutes les fractures étaient déplacées dont 3 cas d’ouverture cutanée. Il y avait 66,1\% (39 cas) de fractures diaphysaires dont 53,57\% localisées au tiers moyen. Sur les 33,9\% (20 cas) de fracture de l'extrémité inférieure, les fractures décollements épiphysaires ont représenté 13 cas dont 7 de type II de Salter et Harris.

Les indications étaient: fracture du fémur survenue dans le cadre d'un polytraumatisme (4 cas), patients de plus de 11 ans (40 cas), échec du traitement orthopédique (2 patients), fracture décollement épiphysaire de l'extrémité inférieure du fémur après échec du traitement orthopédique (chez 12 patients) et une fracture décollement épiphysaire type 4 de Salter et Harris où la chirurgie a été d'emblée indiquée (1 cas). Chez 10 patients traités par ECMES, ont reçu en complément une attelle plâtrée cruropédieuse.

Le délai moyen de prise en charge chirurgicale était de 32 jours, avec des extrêmes de 15 h et 115 jours. En attente du traitement chirurgical définitif, 76,27\% (47 patients) étaient en traction collée sur attelle de Boppe et les autres sous attelle plâtrée. La répartition selon les techniques d’ostéosynthèse utilisées est présentée dans le tableau I.

Les figures 1 et 2 montrent les résultats post opératoires immédiats chez certains opérés par ECMES et par brochage en croix.

La durée moyenne d'hospitalisation était de 45,16 jours. Trois patients avaient présenté une raideur du genou qui s'est vite résolue après rééducation. Avec un recul moyen de 4,3 mois, 32 patients ont été revus. On notait un bon cal osseux sans complication particulière. L'ablation du matériel d'ostéosynthèse a été réalisée chez 38 patients soit 64,4\% des cas. Le délai moyen de cette ablation était de 8 mois.

\section{Discussion}

En traumatologie infantile, les fractures du fémur sont fréquentes et viennent au troisième rang des lésions squelettiques chez l'enfant (ASLANI, 2013). Le profil épidémiologique qui se dégage de notre étude montre que ces lésions touchent plus les garçons (sex-ratio (H-F) de 1,36), de la tranche d’âge de 6 à 11 ans, au décours des accidents de la voie publique. Plusieurs études ont tenté d'expliquer ce profil. En effet, on pense qu’il s’agit de la 
tranche d'âge où les enfants sont de plus en plus autonomes dans leur mobilité, veulent essayer tout et échappent au contrôle des parents augmentant ainsi le risque de survenue d'accidents (ODÉHOURI, 2011). La prédominance masculine serait due à la turbulence des garçons à cette période de la vie et la brutalité de leurs jeux. De plus les filles font l'objet d'une plus grande surveillance parentale les contraignant souvent à rester à la maison, ce qui les préserve ainsi des jeux dangereux et des accidents de la voie publique (SAWADOGO, 2013).

Au plan lésionnel, toutes les études rapportent que parmi les fractures du fémur, ce sont les fractures diaphysaires qui se rencontrent le plus. D’après Loder R.T. et al. (LODER, 2006), elles représentent 1-2\% de toutes les fractures de l'enfant et 20\% des hospitalisations pour Akakpo Numado et al. (AKAKPO-NUMADO, 2014).

Le délai de prise en charge rapporté par les études, se situe généralement dans la première semaine (THOMAS, 2009). Dans notre étude, le délai moyen entre le traumatisme et la prise en charge chirurgicale était de 32 jours. Ce grand retard de la prise en charge dans notre série est le fait d'une longue durée du traitement d'attente, lié au manque de moyens financiers des parents. Ces parents doivent acheter tout le matériel médical (implants et matériel de chirurgie et d'anesthésie) de même qu'ils doivent honorer les frais du bilan préopératoire et payer l'acte opératoire. Ces parents très pauvres, le plus souvent avec un SMIG de 35000 FCFA n'arrivent pas à honorer les dépenses. Ce retard de la prise en charge entraîne :

- l'impossibilité de réaliser une ostéosynthèse à foyer fermé du fait d'un cal qui empêche la mobilisation des fragments pour l'obtention d'une réduction,

- une longue éviction scolaire,

- l'arrêt des activités des parents qui doivent rester auprès de leur enfant à l'hôpital.

Même si jusqu'aux trois dernières décennies la prise en charge des fractures de l'enfant a évolué avec le mode de vie, la technologie des implants et les exigences de la société (KOSUGE, 2015), pour les fractures du fémur, le consensus est établit sur l'indication du traitement orthopédique avant l'âge de 6 ans (RAMSEIER, 2010 ; COLLIN, 2013). Après 6 ans, le traitement chirurgical est plus souvent indiqué (AKAKPO-NUMADO, 2014 ; COLLIN, 2013), afin de réduire les délais d'absence à l'école, la durée du séjour hospitalier et les coûts des soins. Le choix du type d'ostéosynthèse varie alors suivant les équipes et les contextes. La plupart des auteurs préconisent l'ECMES pour traiter les fractures diaphysaires car, réalisé à foyer fermé, il respecte l'hématome périfracturaire, préserve le périoste et la vascularisation locale, et les propriétés biomécaniques de l’os 
en croissance. L'ECMES est de nos jours devenu la technique standard chez les adolescents et les grands enfants (COLLIN, 2013).

Nous avons majoritairement fait usage de l'ECMES dans cette étude mais à foyer ouvert, en raison d'une longue durée du traitement d'attente entrainant la formation de cals qui n'autorisent plus la réduction à foyer fermé. L’inadaptation de la table orthopédique aux enfants et l'indisponibilité de l'amplificateur de brillance sont les raisons supplémentaires de ce choix.

L’ostéosynthèse par plaque a été la deuxième méthode de fixation la plus utilisée dans notre étude chez les grands enfants (18,6\%). Même si en dehors de ses complications classiques, il n'est pas rare d'avoir la défaillance de matériel et les fractures itératives après l'ablation de matériel, cette méthode demeure sûre et efficace (COLLIN, 2013).

Les fractures du fémur sont, dans l'ensemble, de pronostic favorable. Les complications nécessitant une reprise sont peu fréquentes et les séquelles, exceptionnelles.

\section{Conclusion}

Les méthodes de traitement chirurgical les plus employées sont l'ECMES à foyer ouvert en raison de la longue durée d'attente des patients liée aux moyens financiers très limités. Cette méthode présente des avantages certains et sa pratique devra être encouragée dans nos milieux en améliorant le plateau technique et les conditions de vie de la population.

\section{References:}

ASLANI H, TABRIZI A, SADIGHI A, MIRBOLOOK AR. (2013). Treatment of pediatric open femoral fractures with external fixator versus flexible intramedullary nails. Arch Bone Surg. 1(2):64-7.

CAPRA L, LEVIN AV, HOWARD A, SHOULDICE M. (2013). Characteristics of femur fractures in ambulatory young children. Emerg Med J. 30(9):749-753.

CHIGOT P, ESTEVE P. (1967). Traumatologie infantile. Paris: Expansion scientifique française : 427p.

RAMSEIER LE, JANICKI JA, WEIR S, NARAYANAN UG. (2010). Femoral fractures in adolescents: a comparison of four methods of fixation. $J$ Bone Joint Surg Am. 92(5):1122-9.

AKAKPO-NUMADO KG, BOUMÉ AM, SAKIYÈ A, MIHLEUDOAGBOLAN K, GNASINGBE K, TÉKOU H. (2014). Elastic stable intramedullary nailing of femoral shaft fractures in children: particularities and results at Sylvanus Olympio Teaching Hospital of Lomé, Togo. Afr $J$ Paediatr Surg. 11(1):8-11. 
PARSCH KD. (1997). Modern trends in internal fixation of femoral shaft fractures in children. A critical review. J Pediatr Orthop B ;6:117-25.

KREGOR PJ, SONG KM, ROUTT ML, SANGEORZAN BJ, LIDDELL RM, HANSEN ST. (1993). Plate fixation of femoral shaft fractures in multiply injured children. J Bone Joint Surg Am;75:1774-80.

LIGIER JN, METAIZEAU JP, PRÉVOT J, LASCOMBES P. (1998). Elastic stable intramedullary nailing of femoral shaft fractures in children. $J$ Bone Joint Surg $\mathrm{Br}$;70:74-7.

ODÉHOURI K, GOULI J, YAO K, TEMBÉLY S, OUATTARA O, DICK K. (2011). Elastic stable intramedullary nailing in paediatric traumatology at Yopougon Teaching Hospital (Abidjan). Afr J Pediatr Surg; 8: 155-4.

SAWADOGO M, OUEDRAOGO I, KABORE F, BARA J, BANDRE E, WANDAOGO A. (2013). Fractures de la diaphyse fémorale chez l'enfant à Ouagadougou (Burkina faso): aspects épidémiologiques, cliniques et thérapeutiques. Tun Orthop; 89: 599-604.

LODER R.T., O'DONNEL P.W., FINBERG J.R. (2006). Epidemiology and mechanism of femur fracture in children. J Pediatr Orthop. 2006;26(5):561566

THOMAS O, RUBIO A, LEROUX J, LUSAKISIMO S, HAYEK T, GRIFFET J. (2009). Early reduction versus skin traction in the orthopaedic treatment of femoral shaft fractures in children under 6 years old. $J$ Child Orthop; 3: 209-215.

KOSUGE D, BARRY M. (2015). Changing trends in the management of children's fractures. Bone Joint J. 97-B(4):442-8

COLLIN MAY YI-MENG YEN. (2013). Complications of plate fixation of femoral shaft fractures in children and adolescents. J Child Orthop 7:235243.

Tableau I : Répartition des patients selon la technique d'ostéosynthèse utilisée.

\begin{tabular}{c|c|c}
\hline & Nombre de cas & Pourcentage \\
\hline ECMES & 39 & 66,1 \\
Ostéosynthèse par plaque vissée & 11 & 18,6 \\
Brochage & 7 & 11,9 \\
Vissage & 2 & 3,4 \\
Total & 59 & 100 \\
\hline
\end{tabular}

ECMES : Embrochage Centromédullaire Elastique Stable 


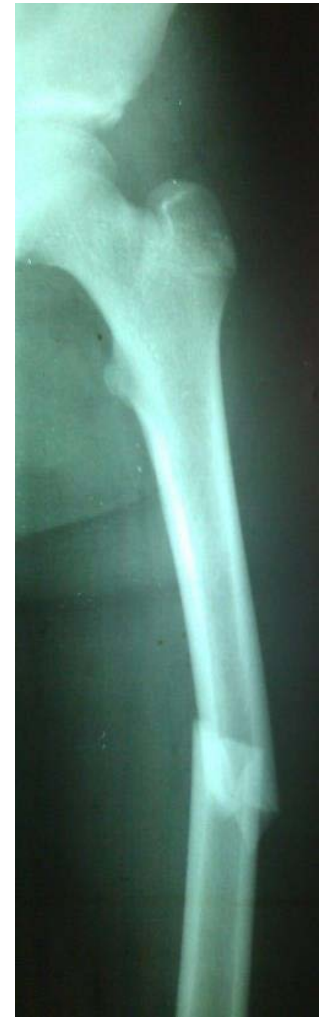

1a

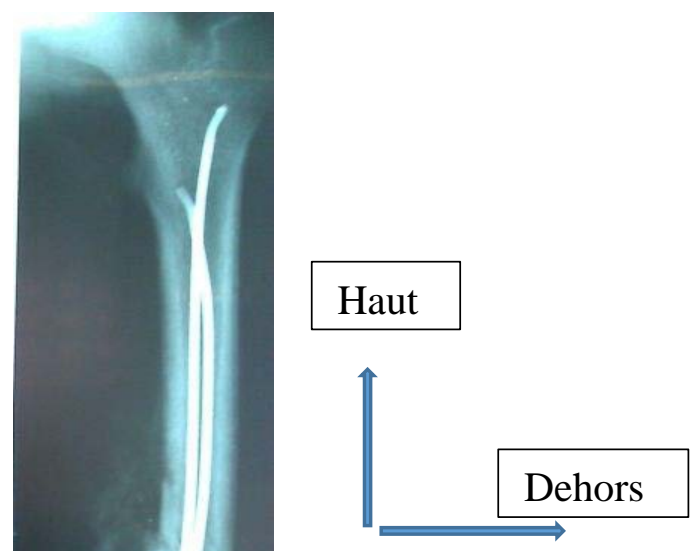

Figure 1a) : Cliché de la radiographique standard de face objectivant une fracture au 1/3 moyen de la diaphyse fémorale gauche chez un enfant de 11 ans.

Figure 1b) : Cliché de la radiographie standard de contrôle de face après réduction et ECMES ascendant. 

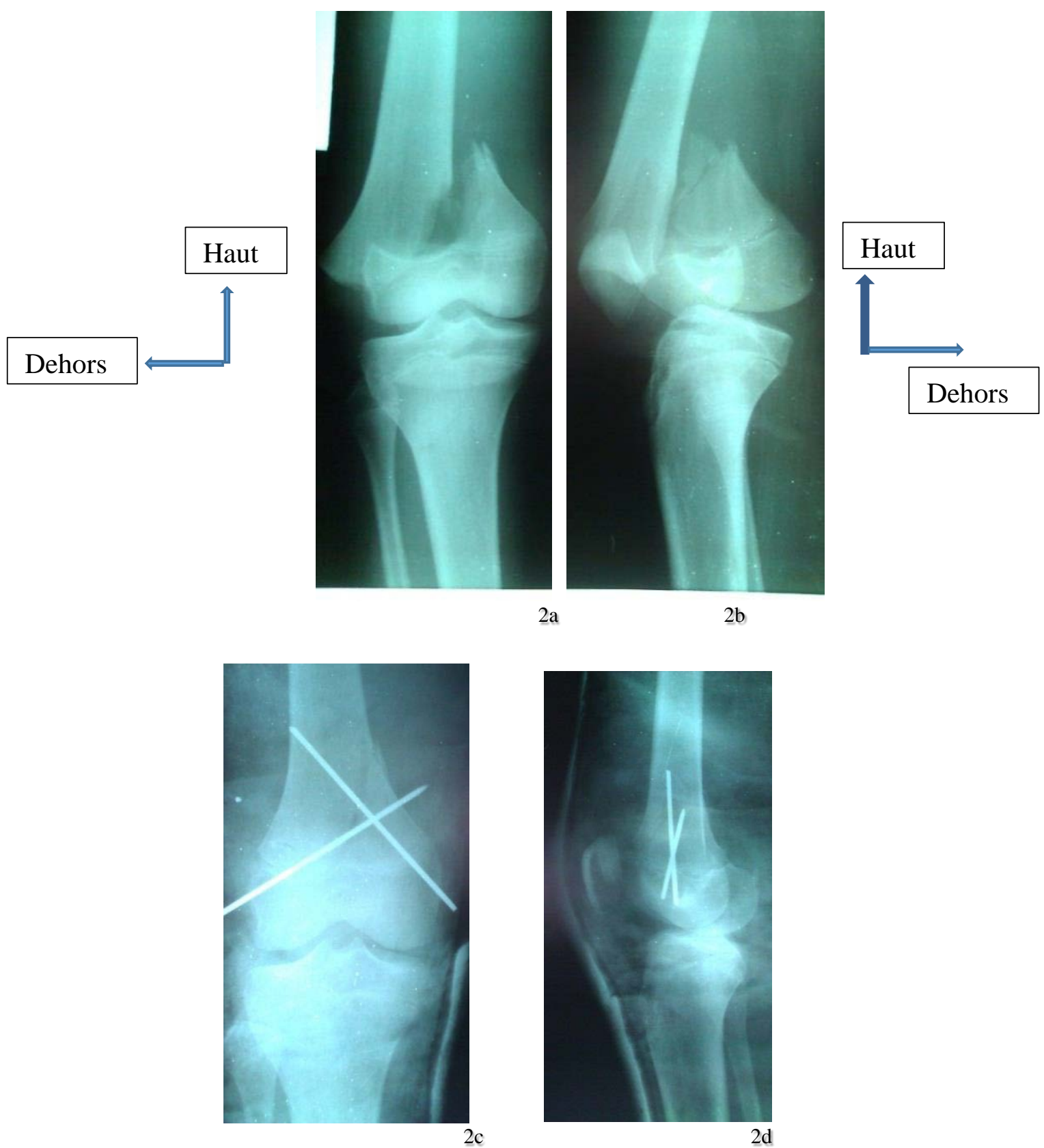

Figure 2 : Radiographies de genou montrant une fracture décollement épiphysaire type II de Salter et

Harris de l'extrémité inférieure du fémur droit chez un enfant de 14 ans (avant la chirurgie) et radiographies de genou de contrôle de face (2c) et de profil (2d) après brochage en croix complété par pâtre. 